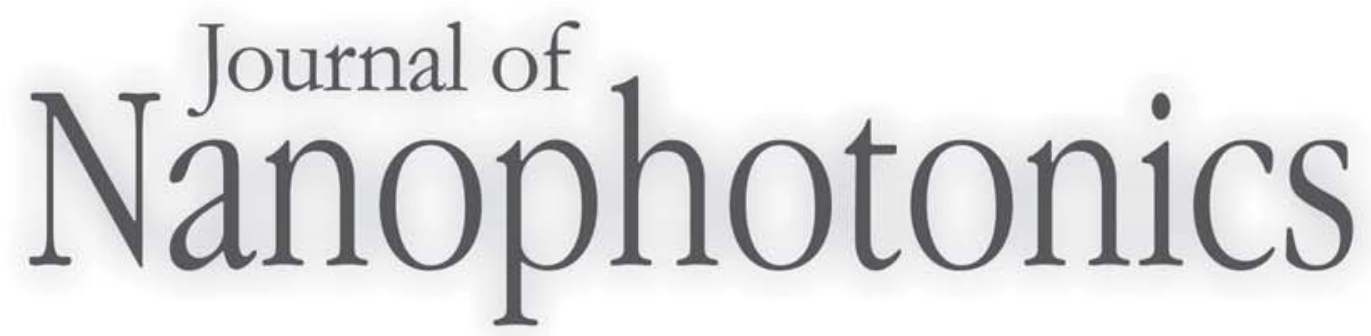

SPIEDigitalLibrary.org/jnp

\title{
Commentary: Nano- and micromachining using laser plasma soft X-rays
}

Tetsuya Makimura

Shuichi Torii

Hiroyuki Niino

Kouichi Murakamia 


\title{
Commentary: Nano- and micromachining using laser plasma soft $\mathrm{X}$-rays
}

\section{Tetsuya Makimura, ${ }^{a}$ Shuichi Torii, ${ }^{a}$ Hiroyuki Niino, ${ }^{b}$ and Kouichi Murakami ${ }^{a}$}

\author{
University of Tsukuba, Institute of Applied Physics, \\ 1-1-1 Ten'nodai, Tsukuba, Ibaraki 305-8573, Japan \\ makumura@bk.tsukuba.ac.jp,bk200820379@s.bk.tsukuba.ac.jp,murakami@bk.tsukuba.ac.jp \\ National Institute of Advanced Industrial Science and Technology, Photonics Research \\ Institute, Central 5, 1-1-1 Higashi, Tsukuba, Ibaraki 305-8565, Japan \\ niino.hiro@aist.go.jp
}

Investigating interactions of intense soft X-rays with solid surfaces and resulting phenomena is still challenging. One could expect material removal from the surfaces (ablation) caused by soft X-ray irradiation at a precision as high as the diffraction limit of the soft X-rays. The X-ray ablation technique could enable us to fabricate nano- and micro-structures on sufaces of even transparent materials such as silica glass, polymethylmethacrylate (PMMA) and polydimethylsiloxane (PDMS), which are highly valued for their use in the fields of nanometric chemical analysis and chemical reactions in medicine and biotechnology.

For machining at high resolution, light with a short wavelength is preferable because of the diffraction limit. In the X-ray region, however, most of the materials have less optical absorption at shorter wavelengths, resulting in no energy transfer of the light to the materials, that is, no ablation. Therefore, photons in the range of $100 \mathrm{eV}-1 \mathrm{keV}$ are suitable for micromachining. So far, synchrotrons have been utilized for extensive studies on interaction of soft X-rays with inorganic materals such as silica glass. However, silica surfaces are etched at a low rate and the exposed surfaces are modified to Si-rich ones. In contrast, photo-etching of polymers has been realized. PMMA surfaces can be removed (etched) by X-ray irradiation without further chemical treatment. PMMA can also be etched by chemicals after X-ray irradiation, which is applied to fabricate micro-molds of PMMA and metals in the Lithographie Galvanoformung und Abformung (LIGA) process. In addition to synchrotrons radiation, soft X-rays from laserproduced plasma have been applied to PMMA ablation [1,2]. Barkusky et al. irradiated PMMA with focused laser plasma soft X-rays (LPSXs), using a Schwaltzschild objective that reflects $\mathrm{X}$-rays at $13.5 \pm 1.0$. Although they achieved high energy as high as $1 \mathrm{~J} / \mathrm{cm}^{2}, \mathrm{X}$-rays out of the band were just absorbed by the objective.

In order to achieve a practical nano- and micromachining technique using LPSXs, we have developed a LPSX source and a focusing mirror for LPSX irradiation at high power density, and have investigated interactions of LPSXs with silica glass $\left(\mathrm{SiO}_{2}\right)$, PMMA, and PDMS at intensities beyond ablation threshold. Figure 1(a) shows the experimental setup. Tantalum plasma was generated by irradiation of a Ta target $(T)$ with $532 \mathrm{~nm} \mathrm{Nd:YAG} \mathrm{laser} \mathrm{light}(Y)$ with a pulse duration of $10 \mathrm{~ns}$ and a pulse energy of 500-800 mJ/pulse. The Nd:YAG laser was operated at $10 \mathrm{~Hz}$ and a single pulse was selected using a mechanical shutter. The laserproduced Ta plasma emits soft X-rays $(X)$ around $100 \mathrm{eV}(\simeq 10 \mathrm{~nm})$, as shown in Fig. 1(b). The LPSX generation was carried out in a vacuum chamber (VIC International Inc.) at $2 \times 10^{-4} \mathrm{~Pa}$ in order to avoid aborption of the generated LPSXs by air. The LPSXs have the same pulse duration as that of the Nd:YAG laser light. The LPSXs were focused onto a surface of silica $\left(\mathrm{SiO}_{2}\right)$ glass plates $(S)$ using an ellipsoidal mirror $(M)$ (Hidaka Kougaku Kenkyusho Co., Ltd.) that is made of silica glass and is coated with a Au layer on top of it. The mirror is designed so as to maximize power density of LPSXs at $100 \mathrm{eV}$ on the surface of the samples [3]. As shown in Fig. 1(c), silica glass has optical absorption in the photon energy region. The details of the

(C)2010 Society of Photo-Optical Instrumentation Engineers [DOI: 10.1117/1.3488615] 
(a)

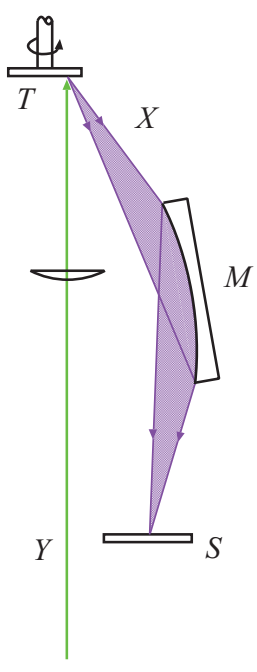

Wavelength (nm)

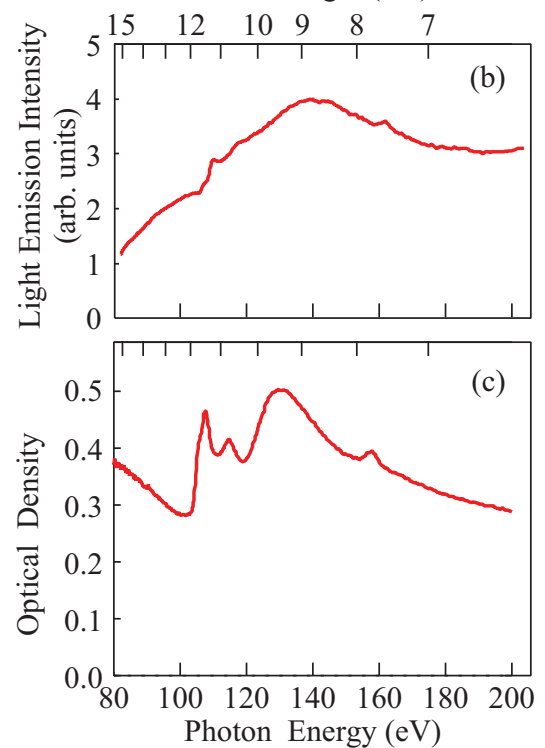

Fig. 1. (a) Experimental setup for nano- and micromachining using laser plasma soft X-rays. (b) X-ray spectrum of light emission from laser-produced Ta plasma. (c) Absorption spectrum of $\mathrm{a} \mathrm{SiO}_{2}$ film with a thickness of $100 \mathrm{~nm}$.

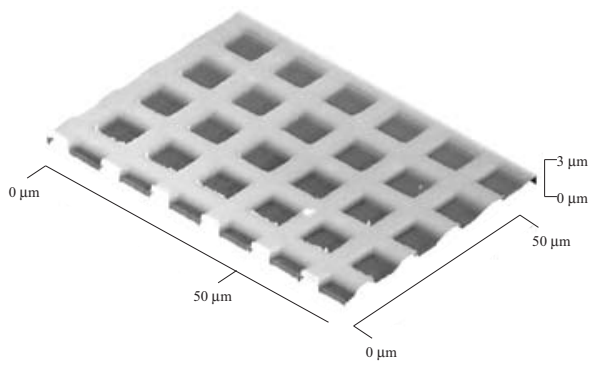

(a)

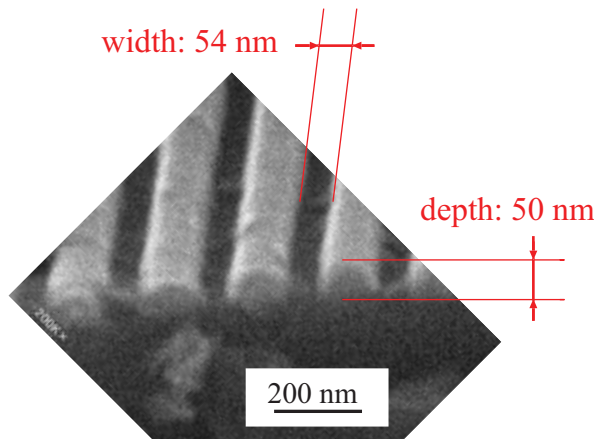

(b)

Fig. 2. Surface profiles of silica glass after laser plasma soft X-ray irradiation though (a) a Ni contact mask with square apertures with a window width of $8 \mu \mathrm{m}$ and (b) a WSi contact mask with a line and space pattern with a window width of $53 \mathrm{~nm}$.

soft X-ray spectroscopy are given elsewhere $[4,5]$. The power density of LPSX was roughly estimated to be $1 \times 10^{8} \mathrm{~W} / \mathrm{cm}^{2}$ from (a) conversion efficiency from pulsed Nd:YAG laser light at $532 \mathrm{~nm}$ to soft X-rays [6], (b) focusing efficiency [3,7], and (c) area of cross section of LPSX beam on the samples, which was determined by geometry of the ellipsoidal mirror and samples [3]. The power density can be adjusted by keeping the samples away from the focal point of the LPSX beam.

Figure 2(a) shows a confocal laser microscope (Keyence, VK-8510) image of silica glass after LPSX irradiation through a Ni contact mask with square apertures with a width of $8 \mu \mathrm{m}$. The LPSX-irradiated regions are removed and square holes are observed. The surface is ablated at $47 \mathrm{~nm} / \mathrm{shot}$ at $3 \times 10^{7} \mathrm{~W} / \mathrm{cm}^{2}$ and surface roughness $R_{\mathrm{a}}$ is $1 \mathrm{~nm}$ after 10 shots of LPSX irradiation. The ablation depth is proportional to shot number of LPSX irradiation at rates of $0.1-150 \mathrm{~nm} / \mathrm{shot}$. It is remarkable that high-quality micromachining at high rates can be achieved using the LPSX technique. 
In order to fabricate nanostructures on silica glass, a WSi line-and-space mask was fabricated on a silica plate by the electron beam lithography technique. The WSi mask had windows with a width of $53 \mathrm{~nm}$ and a ptich of $175 \mathrm{~nm}$. After LPSX irradiation through the windows of the WSi contact mask, the WSi mask was removed by the selective ion etching technique. Figure 2(b) shows a cross section of the nano-trenches fabricated on the silica glass plate, observed using a scanning electron microscope. We have demonstrated that nanostructures can be fabricated by the LPSX technique.

The thermal diffusion length during the 10-ns LPSX pulse is estimated to be $80 \mathrm{~nm}$ while the fabricated nanostructures have finer features. The result indicates that ablation from a silica surface occurs faster than the thermal diffusion. In order to clarify the ablation process, we performed mass spectroscopy for species ejected from silica surface by LPSX irradiation [8]. We found that silica glass is broken into atomic species and that $1-15 \%$ of the species are ionized. Most of the ions are generated during LPSX irradiation before ejection from silica surface, because ionization caused by interactions with photons in the soft X-ray region in the gas phase is almost prohibited. Therefore, the ablation is possibly caused by Coulomb repulsion of ionic species that are generated by LPSX irradiation. This enables us to fabricate nanostructures on silica surfaces using even nanosecond LPSX pulses. In the infrared, visible and ultravilet regions, lasers with shorter pulse durations are often requied to realize micromachining at higher resolution as the resolution is limited by thermal diffusion lengths during laser pulses.

Recently, we found that silica glass can be ablated using LPSX in a narrow range of 6$25 \mathrm{~nm}$. The LPSX was obtained using a Zr filter with a thickness of $100 \mathrm{~nm}$ that is transparent in the chosen spectral region. Compared to the wide band LPSX without any Zr filter, the narrow band LPSXs have an advantage that higher resolution can be achieved during LPSX irradiation. Further, it is essential to irradiate materials with LPSXs at sufficiently high power densities for the purpose of micromachining. Hence, it is advantageous to use X-ray optics with a glancing angle of incident such as an ellipsoidal mirror. The optics with glacing angle can focus LPSXs in a wide photon-energy range. Imaging optics with glancing angles such as a Wolter mirror would be preferable for practical use.

In addition to silica glass $\left(\mathrm{SiO}_{2}\right)$, the micromachining technique using LPSXs is applicable to a variety of materials such as Pyrex and $\mathrm{Al}_{2} \mathrm{O}_{3}$, PMMA [9] and PDMS. By applying the PMMA ablation using LPSXs, syncrotrons could be replaced by laser-produced plasma sources in the LIGA process. This would enable us to fabricate micro components made of polymers and metals in a practical way. The PDMS ablation could be applied to medicine and biotechnology because of its biocompatiblity. Micro-structures at high aspect ratio such as through-holes could be fabricated using LPSXs.

In conclusion, we have investigated a practical nano- and micromachining using LPSXs. It is found that silica glass is ablated at rates of $0.2-150 \mathrm{~nm} / \mathrm{shot}$. At the same time, high quality micromachining can be achieved. Typically, suraface roughness $R_{\mathrm{a}}$ is $1 \mathrm{~nm}$ after 10 shots of LPSX ablation at a rate of $47 \mathrm{~nm} / \mathrm{shot}$. Furthermore, it is demonstrated that $50 \mathrm{~nm}$ trenches are fabricated using LPSXs. The LPSX technique would provide a practical way for fabricating micro total analysis systems, micro fludic devices, MEMS devices.

\section{Acknowledgments}

This work was partially supported by Nippon Sheet Glass Foundation for Materials Science and Engineering, Amada foundation for metal work technology, Industrial Technology Research Grant Program in 2001-2005 from NEDO of Japan and JSPS KAKENHI (15656183, 17360348, 22360016). 


\section{References}

[1] H. Fiedorowicz, A. Bartnik, M. Bittner, L. J. J. Krasa, P. Kubat, J. Mikolajczyk, and R. Rakowski, "Micromachining of organic polymers by direct photo-etching using a laser plasma x-ray source," Microelectron. Eng. 73-74, 336-339 (2004) [doi:10.1016/j.mee.2004.02.063].

[2] F. Barkusky, A. Bayer, C. Peth, and K. Mann, "Direct photoetching of polymers using radiation of high energy density from a table-top extreme ultraviolet plasma source," J. of Appl. Phys. 105, 014906 (2009) [doi:10.1063/1.3054565].

[3] T. Makimura, H. Miyamoto, Y. Kenmotsu, , K. Murakami, and H. Niino, "Direct micromachining of quartz glass plates using pulsed laser plasma soft x-rays," Appl. Phys. Lett. 86, 103111 (2005) [doi:10.1063/1.1882750].

[4] T. Makimura and K. Murakami, "Dynamics of silicon plume generated by laser ablation and its chemical reaction," Appl. Surf. Sci. 96-98, 242-250 (1996) [doi:10.1016/01694332(95)00428-9].

[5] T. Makimura, T. Sakuramoto, and K. Murakami, "Time-resolved X-ray absorption spectroscopy for laser-ablated silicon particles in xenon gas," Jpn. J. Appl. Phys. 35, L735-L737 (1996) [doi:10.1143/JJAP.35.L735].

[6] R. C. Spitzer, T. J. Orzechowski, D. W. Phillion, R. L. Kauffmann, and C. Cerjan, "Conversion efficiencies from laser-produced plasmas in the extreme ultraviolet regime," J. Appl. Phys. 79, 2251-2258 (1996) [doi:10.1063/1.361149].

[7] B. L. Henke, E. M. Gullikson, and J. C. Davis, "X-ray interactions: Photoabsorption, scattering, transmission, and reflection at $e=50-30,000 \mathrm{eV}, z=1-92$," At. Data Nucl. Data Tables 54, 181-342 (1993) [doi:10.1006/adnd.1993.1013].

[8] S. Torii, T. Fujimori, T. Makimura, H. Niino, and K. Murakami, "Ablation process of silica glass induced by laser plasma soft x-ray irradiation,” Appl. Surf. Sci. 255, 9840-9842 (2009) [doi:10.1016/j.apsusc.2009.04.102].

[9] S. Torii, T. Makimura, K. Okazaki, D. Nakamura, A. Takahashi, T. Okada, H. Niino, and K. Murakami, "Direct etching of poly(methyl methacrylate) using laser plasma soft x-rays," Appl. Phys. Exp. 3, 066502 (2010) [doi:10.1143/APEX.3.066502]. 